\title{
Q0045-3337: models including strong lensing by a spiral galaxy
}

\author{
M. Chieregato ${ }^{1,2}$, M. Miranda ${ }^{1}$, and P. Jetzer ${ }^{1}$ \\ 1 Institut für Theoretische Physik der Universität Zürich, Winterthurerstrasse 190, 8057 Zürich, Switzerland \\ e-mail: [matteo; solar; jetzer]@physik.unizh.ch \\ 2 Dipartimento di Fisica e Matematica, Università dell'Insubria, via Valleggio 11, 22100 Como, Italy
}

Received 29 November 2006 / Accepted 9 July 2007

\section{ABSTRACT}

\begin{abstract}
Aims. Falomo et al. (2005, A\&A, 434, 469) discovered a disk-like galaxy at $\sim 1.2$ arcsec from the QSO Q0045-3337 by means of ESO VLT adaptive optics. They estimated a galaxy Einstein radius (for a point mass) of comparable size, thus suggesting the existence of a new, rare, spiral lens candidate, despite no evident image splitting. We discuss the possible lensing effect of the galaxy in more detail. Methods. We performed two-dimensional surface photometry on the VLT image of the galaxy, confirming its spiral nature. We then verified if simple mass models, partially constrained by observational data, require unrealistic parameters to produce a still hidden second quasar image. We also evaluated the viability of an instrumental or a lensing origin of the observed QSO deformation.

Results. After galaxy model subtraction, we found a residual image, which we consider unlikely to be related to gravitational lensing. Existing data are not sufficient to assess the presence of image splitting, nor to constrain the number of images or discriminate between galaxy mass models.

Conclusions. Further observations are mandatory to progress in the study of this remarkable system, which could shed more light on the lensing behavior of spiral galaxies.
\end{abstract}

Key words. gravitational lensing - galaxies: spiral - quasars: individual: Q0045-3337

\section{Introduction}

The number of known strong lensed quasars increased significantly in the last few years to the current value of $\sim 90$ (Kochanek et al. 2004; CASTLES web site, http://cfa-www.harvard.edu/castles/). The reason for this increase is mainly the availability of new observational facilities, in particular the Hubble Space Telescope, of dedicated surveys (CLASS, Myers et al. 2003; Browne et al. 2003) and of large public observational databases, such as the FIRST (White et al. 2000) and in particular the Sloan Digital Sky Survey (York et al. 2000; Adelman-McCarthy et al. 2006).

The lion's share of the lens population is made up of early-type galaxies. In fact, up to now, only 5 systems have been confidently identified as having spiral galaxies: firstly, the Einstein cross, Q2237+0305 (Huchra et al. 1985), then B1600+434 (Jackson et al. 1995), PMNJ2004-1349 (Winn et al. 2003), B0218+357 (Patnaik et al. 1993) and PKS 1830-211 (Pramesh Rao \& Subrahmanyan 1988). Each of these systems has its own peculiarities (inclination of the lens galaxy, richness of the lens environment, etc.), making it unique, and can therefore bring its own valuable contribution to the knowledge of spiral galaxies mass distribution.

The discovery of a late-type galaxy at $\sim 1.2^{\prime \prime}$ from Q00453337 (Falomo et al. 2005) suggested the existence of another candidate. In fact, while there is no evident image splitting, Falomo et al. inferred a galaxy Einstein radius of size comparable to the distance between the galaxy and the QSO, under reasonable hypotheses on galaxy redshift and mass-to-light ratio, and considering the galaxy as a point mass.

Existing, limited data hamper detailed modeling of Q00453337. We investigated some simple configurations compatible with the known properties of the system, in the hope of restricting the range of possibilities and to focus future observations.

\section{Observational parameters}

Q0045-3337 ( $\mathrm{RA}=004741.85$, Dec $=-3320$ 55.1) is a radio quiet quasar with a reported redshift of $z=2.14$ (Iovino et al. 1996) and a $V$ magnitude of 18.75 (Véron-Cetty \& Véron 2001). The NAOS-CONICA VLT $K$ band image of Q0045-3337 (Falomo et al. 2005) revealed a galaxy at 1.14" SE from the quasar (A and B in Fig. 1). The $K$ magnitude of the QSO is 17.80, while the one of the galaxy is 16.97 (see the following paragraph for a discussion of the galaxy surface photometry). The only other object seen in the field, apart from the guide star, is source C, with a $K$ magnitude of $20.90,3.5^{\prime \prime} \mathrm{N}$ of A.

The quasar itself appears noticeably elliptical (see Sect. 5.2). There is no redshift measurement for the galaxy. Falomo et al. (2005) estimated a redshift range of 0.4-1 from an educated guess on the galaxy absolute $K$ magnitude ( -24 to -26$)$ and effective radius ( 2 to $3 \mathrm{kpc}$ ).

\section{Surface photometry and galaxy image subtraction}

We performed two-dimensional surface photometry on the NAOS CONICA image of the galaxy with the software GALFIT (version 2.0.3c; Peng et al. 2002). Two-dimensional photometry for adaptive optics is complicated by the peculiarities of the point spread function (PSF). In fact, each extended component of the GALFIT models is usually convolved with the PSF of the image; moreover, the PSF is used to fit point-like sources. It is a well known feature of adaptive optics that the PSF can assume 


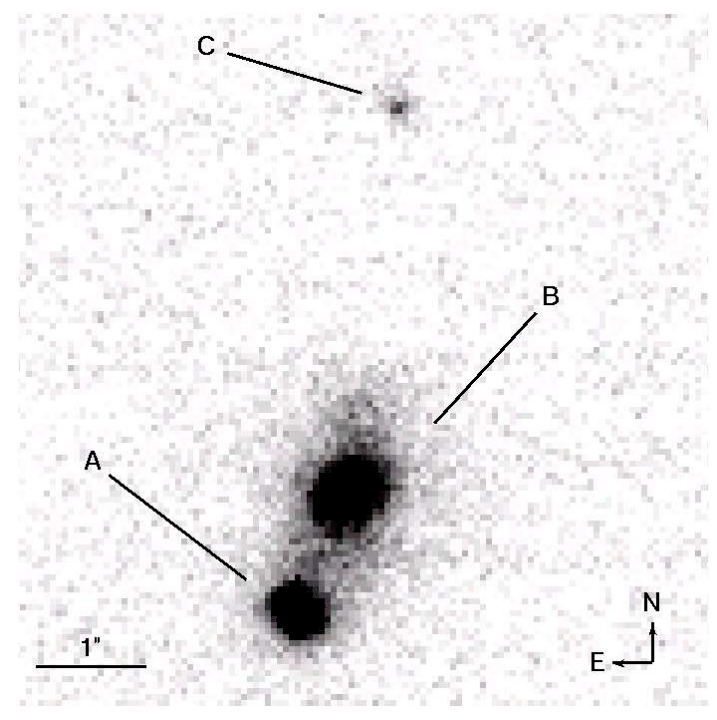

Fig. 1. Q0045-3337 and its foreground galaxy. A close-up of the NAOSCONICA image of Q0045-3337 and its foreground galaxy (see text for details).

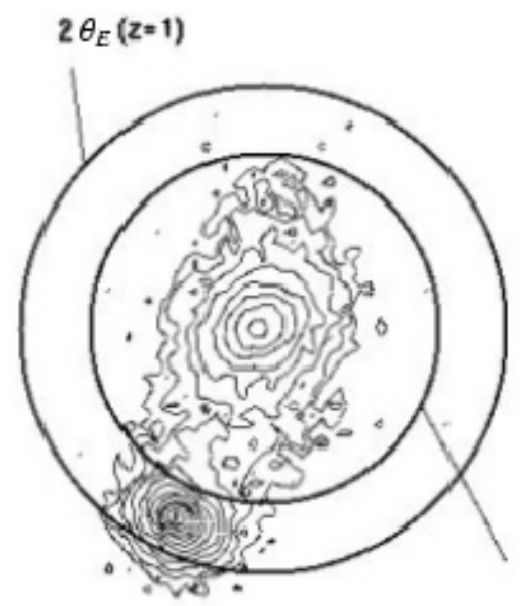

\section{$2 \theta_{E}(z=0.4)$}

Fig. 2. Contour map and Einstein radii. Contour map from the NAOSCONICA image, with superimposed circles of twice the Einstein radius for SIS with dispersion velocities calibrated with the Tully-Fisher law (see text for details).

complex shapes, in particular it usually varies with angular distance from the guide star, and can be elongated in the direction of the guide star. Usually, analytical modeling of the PSF from ancillary data is not possible, though this situation is likely to change in the future (see Clénet et al. 2006, for the NaCo case). What should therefore be done is to infer a PSF template from observations of bright stars at a similar angular distance from the guide star, observations performed just before and just after the observation to be analyzed. Unfortunately, this part of the program was not executed for the NaCo observation of Q0045-3337 (Falomo et al. 2005).

The QSO itself, besides being "contaminated" from light from the galaxy, appears visibly elliptical, and it is not clear if its ellipticity is an effect of PSF elongation or is due to a different cause (Fig. 2; Sect. 5.2). The only other source, object C, is very faint and irregular, and its point-like or extended nature is not well determined.

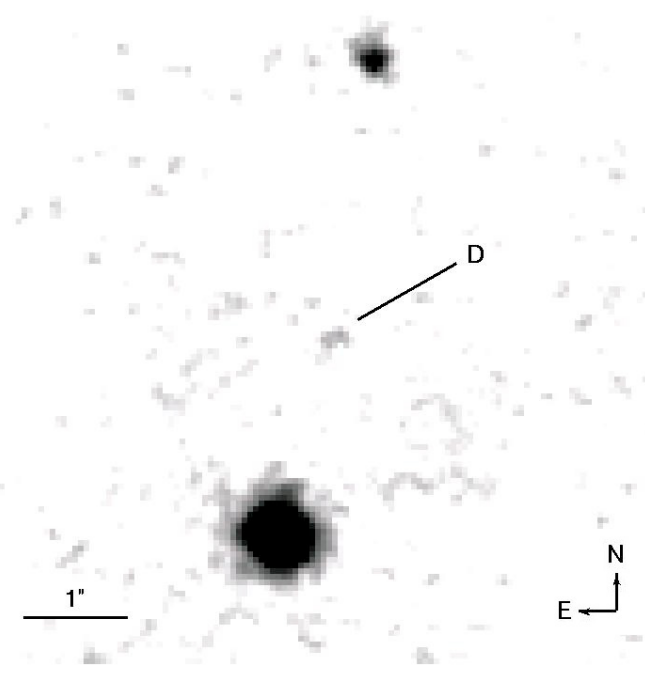

Fig. 3. Q0045-3337 galaxy subtracted. The NAOS-CONICA image of Q0045-3337 after galaxy model subtraction, top-hat smoothing and contrast enhancing, in logarithmic scale. The residual feature (D) is clearly visible midway between object C and QSO.

We therefore performed a simultaneous fit of the galaxy and the QSO without convolving a PSF, using GALFIT models also for the QSO light distribution.

A two component model is required to account for the galaxy light distribution (plus two other components to fit the QSO); in particular, we obtained the best results with two Sérsic models of integrated magnitude 17.19 and 18.80 , both with Sérsic index 0.63 ( 1 is the exponential disk, 0.5 the Gaussian) and $R_{\mathrm{e}}$ respectively $13.5^{\prime \prime}$ and $3.4^{\prime \prime}$. The inner model is tilted by 50 degrees with respect to the outer.

After galaxy model subtraction, the main residual feature is an irregularly shaped object at $0.85^{\prime \prime} \mathrm{N}$ of the galaxy centroid ( $\sim 2^{\prime \prime}$ from the QSO; Fig. 3). The residual is very elongated, but due to its faintness ( $K \sim 22.6$, poorly constrained) it is not possible to firmly establish its point-like or extended nature.

We checked the robustness of our results using PSF templates obtained from the QSO, keeping in mind the already mentioned caveat. In particular, we used both the background subtracted QSO cropped image and the best fit QSO analytical profile (this last case should be less contaminated from galaxy light; however, it has the additional difficulty that the QSO fit is not perfect, with a noticeable residual left).

Even using these PSF templates, the need for two components to account for the galaxy light distribution still stands. In particular, the large difference in orientation between the outer and inner ellipsoids is unchanged.

The residual component is also unchanged. The only appreciable difference is a slight change in the two Sérsic indexes; the inner one becomes steeper (0.71), the outer one shallower (0.57). In order to verify if the residual image could have an explanation in terms of inefficient removal of bad pixels or cosmic rays, we carefully examined each of the frames that compose the summed NACO image. We did not find anything suspicious in any of the frames, and therefore we conclude that such interpretations of the residual image are unlikely.

\section{Single image or multiple images}

The zero-th order question that Q0045-3337 and its foreground galaxy raise is whether the lensing effect is strong, i.e. whether 
there are other, still unseen, QSO images, and eventually where they should be looked for.

In fact, if truly there is no image splitting, only a reduced amount of information can be extracted on the galaxy (e.g. Narayan \& Schneider 1990; Le Brun et al. 2000), though it can be still useful, in our case, to probe the behavior of the strong lensing cross section for a spiral galaxy.

The simplest galaxy lens model with some physical meaning is the singular isothermal sphere (SIS). Within the SIS framework, all lens and source quantities are tied by simple analytical relations. In particular, a necessary and sufficient condition for multiple image formation is that the Einstein radius $\theta_{E}$ has to be greater than half $\theta_{I}$, the distance of the brightest image from the lens $\left(\theta_{E} \geq 1 / 2 \theta_{I}\right.$; Narayan \& Schneider 1990). The Einstein radius is defined as $\theta_{E}=4 \pi \times\left(\sigma_{\mathrm{v}} / c\right)^{2} \times D_{\mathrm{ds}} / D_{\mathrm{s}}$, where $\sigma_{\mathrm{v}}$ is the velocity dispersion of the SIS, $D_{\mathrm{ds}}$ is the angular diameter distances between the source and the lens and $D_{\mathrm{s}}$ is the angular diameter distance between the source and the observer. The $0.57^{\prime \prime}$ discriminating threshold corresponds to a SIS velocity dispersion of $167 \mathrm{~km} \mathrm{~s}^{-1}$ for a lens galaxy redshift of 0.4 , and of $228 \mathrm{~km} \mathrm{~s}^{-1}$ for a redshift of 1 . The mass enclosed in $\theta_{I}$ is respectively $7.8 \times 10^{10} M_{\odot}$ inside $6.1 \mathrm{kpc}$ and $2.2 \times 10^{11} M_{\odot}$ inside $9.1 \mathrm{kpc}$. Since the companion galaxy of Q0045-3337 is a spiral, the Tully-Fisher relationship (Pierini \& Tuffs 1999) can be used to infer a circular velocity range of $218-351 \mathrm{~km} \mathrm{~s}^{-1}$ from the (guessed) absolute magnitude. Under the assumption that the $\sigma_{\mathrm{v}}$ parameter of the SIS mass distribution is $1 / \sqrt{2}$ of the maximum circular velocity, the obtained $\sigma_{\mathrm{v}}$ are $154 \mathrm{~km} \mathrm{~s}^{-1}$ and $248 \mathrm{~km} \mathrm{~s}^{-1}$, and the inferred Einstein radius ranges from $0.49^{\prime \prime}$ to $0.68^{\prime \prime}$ (Fig. 2). Such values do not allow us to confirm or reject image splitting, keeping in mind the intrinsic dispersion in the Tully-Fisher relationship, the possibility of evolutionary effects and in particular the extreme simplification used in assuming a SIS model.

If the lensing is weak, its effects are magnification of point sources and tangential stretching of extended sources. We will discuss the stretching effect later. In the SIS framework the maximum magnification without image splitting is 2 . Much higher values can be reached, at least in principle, with different mass models (Keeton et al. 2005).

\section{Multiple images}

In this section we investigate the hypothesis that additional, yet unseen (or not recognized) images are produced. We slightly complicate the galaxy model abandoning the spherical symmetry. Singular and non-singular Isothermal Ellipsoidal Mass Distribution (SIEMD, PIEMD) and ellipsoidal potential have been studied in some detail over the last $\sim 15$ years (e.g. Kassiola \& Kovner 1993; Schramm 1990; Kormann et al. 1994). The structure of the caustic curves of non-spherical models changes qualitatively, with the appearance of the astroid-shaped tangential caustic. The odd image theorem needs no more infinite demagnification of the central maximum image, and the presence of two caustics allows the production of up to five images. These models should be more realistic than the SIS oversimplification; however, they still lack "on the field" tests for spiral lenses, due to the already mentioned paucity of known cases.

Our goal in this section is not to make a detailed model of the galaxy mass distribution, but only to check if strong, imagesplitting, lensing configurations can be compatible with the constraint that no other image is seen in the NAOS-Conica data, and if that is the case to lead the way for future observations. To
Table 1. GravLens models. Parameters are, from top to bottom, SIEMD Einstein radius parameter, exponential disk central surface density, ellipticity, position angle, shear strength, shear angle and exponential disk elliptical scale radius.

\begin{tabular}{lcccc}
\hline \hline Parameter & Model I & Model II & Model III & Model IV \\
\hline Type & SIEMD & SIEMD & SIEMD $_{\gamma}$ & ExpDisk \\
\hline$R_{E, g a l}{ }^{a}\left({ }^{\prime \prime}\right)$ & 0.86 & 0.49 & 0.86 & - \\
$\kappa_{0}{ }^{c}$ & - & - & - & 2.45 \\
$e^{c}$ & 0.34 & 0.34 & 0.30 & 0.67 \\
$\mathrm{PA}^{d}(\mathrm{deg})$ & -13 & 0 & -5 & -5 \\
$\gamma$ & - & - & 0.06 & - \\
$\phi_{\gamma}{ }^{d}(\mathrm{deg})$ & - & - & -20 & - \\
$R_{\mathrm{d}}\left({ }^{\prime \prime}\right)$ & - & - & - & 0.5 \\
\hline
\end{tabular}

${ }^{a}$ Semiminor axis; ${ }^{b}$ in units of critical density; ${ }^{c}$ defined as $(1-q) / q$, with $q$ axis ratio; ${ }^{d}$ North over East.

reproduce the qualitative behavior of different cases, we extensively used the Java applet SimpLens (Saha \& Williams 2003). Subsequently, we ran some simple simulations with the Gravlens software (Keeton 2001) to verify if the proposed configurations require a non-realistic choice of parameters. Table 1 shows the models used later in the text.

\subsection{Three images}

The easiest, and therefore favored by the Ockham's razor, imagesplitting configuration is a three-image configuration (similar to that shown in Fig. 4, top panels). In this case, the source has to be positioned between the external, radial, caustic and the internal astroid. The maximum, demagnified image is hidden by the lens galaxy bulge. Let us suppose that the residual image found in Sect. 3 is unrelated. If this is the case, the absence of other point sources requires a large magnification ratio between the minimum and the saddle point images. As a working hypothesis we chose a magnification ratio value of 20 as threshold of validity for the models. Even if this threshold would be too low to be compatible with the absence of any visible trace of the saddle point image, there are other ways to boost the magnification ratio. In particular, the saddle point image could be hidden by a strong obscuration if there is enough dust in the galaxy (even if no evidence of a dust lane is found from the surface photometry), or the minimum image flux could be enhanced by substructure lensing, microlensing and by the QSO variability itself.

A large magnification ratio can be obtained with a SIEMD in two ways (see Keeton et al. 2005, for an extensive discussion). First, you can put the source in one of four drop-shaped areas just outside the astroid cusps (Fig. 5; Keeton et al. 2005, Fig. 1). We will refer to this case as the drops configuration. If the source is in one of the two, very small, drops along the minor axis, the saddle point is the brightest image. This does not correspond to our case, and would require an unphysical mass distribution orthogonal to the light distribution.

If instead the source is in one of the two larger drops along the major axis, the minimum image is greatly magnified, and it forms very close to the tangential critical curve; thus the mass scale of the system is roughly fixed by the distance between the minimum image and the galaxy center. Model I of Table 1 is an example of a situation of this kind. The ellipticity is chosen to not differ too much from the light distribution; then the Einstein radius parameter (minor semiaxis of the tangential critical curve) is settled by the distance between the QSO and the galaxy center, and the position angle by the need to stay in the drop area. 

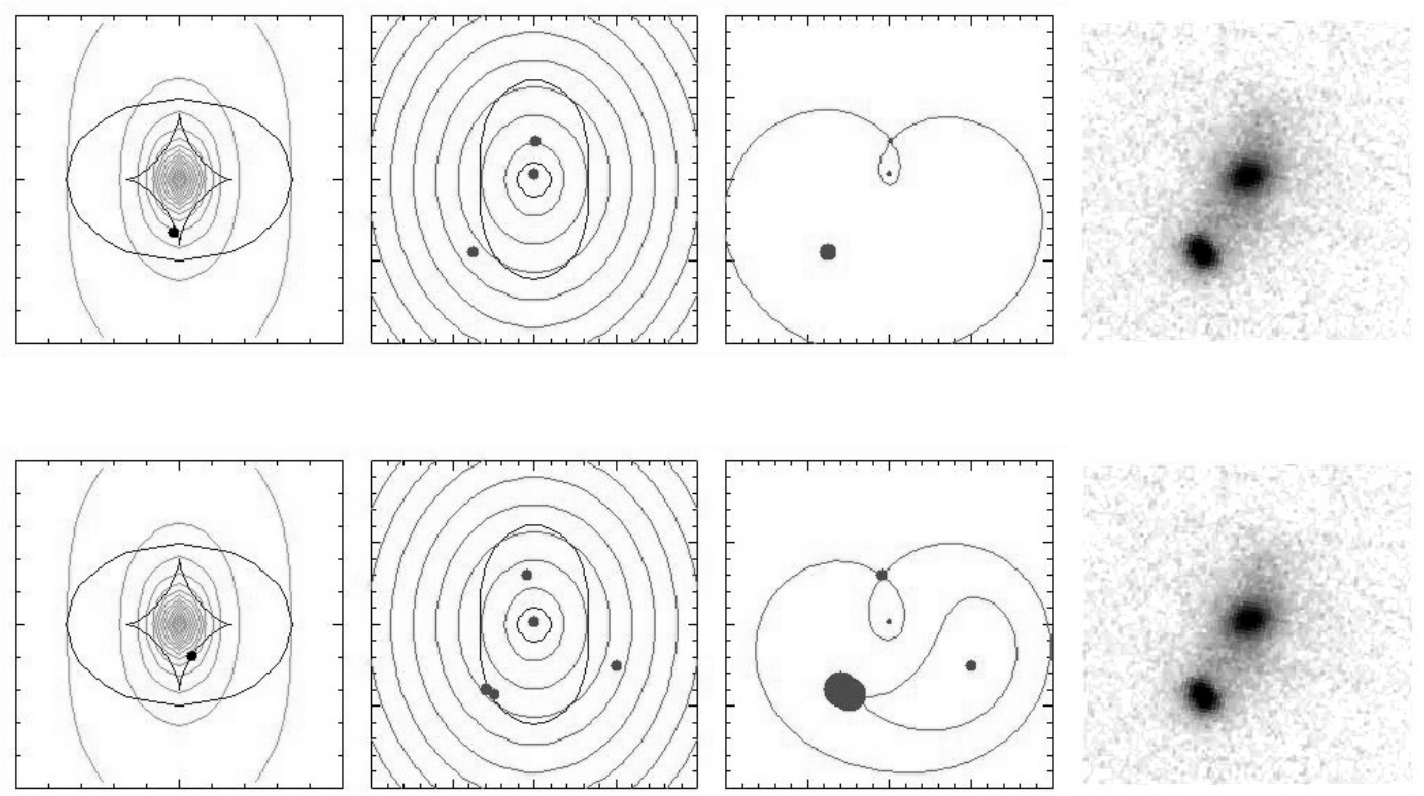

Fig. 4. Lensing scenarios for Q0045-3337. A qualitative representation of lensing scenarios for Q0045-3337. From top to bottom: three-image case with the saddle point image hidden; five-image case with a saddle point image merged with a minimum image, the other saddle point and the other minimum images hidden. From left to right: SimpLens source position (point), isodensity contours (light curves) and caustics (grey curves); SimpLens images positions (points) and critical curves (grey curves); SimpLens image positions (points) with area proportional to the magnification and virtual light-travel time contours (grey curves); Q0045-337 and foreground galaxy, properly rotated and scaled to be directly comparable with the SimpLens images. The SimpLens model used is a non-singular isothermal ellipsoidal potential, with a core radius of 0.01 and an ellipticity of 0.37 .

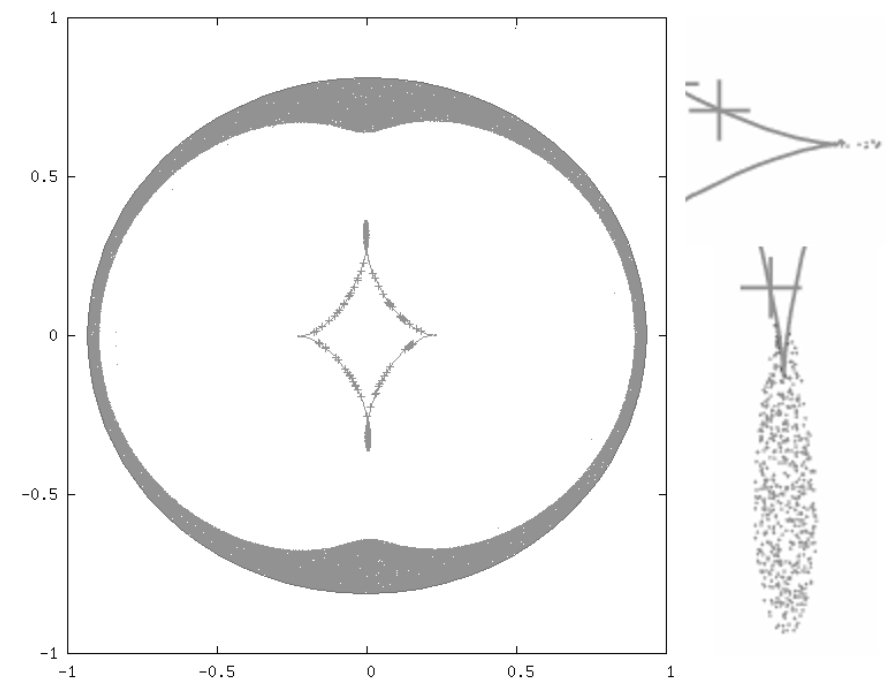

Fig. 5. Simulated sources. Source plane of a SIEMD model (see Table 1 for parameters) with simulated sources. The grey area is the locus of sources producing three images with a magnification ratio $>20$, crosses are sources producing five images with a magnification $>40$. On the right, close-ups of two drop areas. More details are reported in the text.

The other way to obtain a large magnification ratio is to put the source close to the radial caustic, in the external grey area of Fig. 5. We refer to this case as the mouth configuration. In this case, the saddle point image is strongly demagnified. The minimum image forms further away from the tangential critical curve. Therefore, for a fixed distance between the minimum image and the lens center, the mass scale of the system is reduced. The constraint on the position angle is also relaxed. Model II of
Table 1 is an example of this scenario. Note that in this case the Einstein radius parameter is at the lower end of the SIS range of Sect. 4, and the mass distribution is not aligned with either of the light distribution components.

A misalignment between mass and light can be mimicked by the presence of shear, or be due to the aftermath of a major merger; this last hypothesis could be supported by the isophotal twisting and core-disk misalignment of the galaxy. In model III of Table 1 we added a shear component, that can be either due to real shear or just a way to simulate an intrinsic complex angular structure of the galaxy mass distribution. With this model, the production of large magnification ratios is easier, so at our 20 threshold the two areas of Fig. 5 are actually merged (e.g. Keeton et al. 2005, Fig. 1).

Let us consider instead the hypothesis that the residual image is the saddle point image. The elongated shape of the residual seems to contradict this speculation; however, since we are dealing with an object near to the plate limit, obtained after subtraction of an extended component and possibly absorbed, and furthermore we do not have a firmly established PSF shape, the morphological evidence cannot be decisive. In the same way the position and the faintness of the residual are less than conclusive clues against the saddle point image interpretation. The face value magnification ratio between the QSO and the residual is $\sim 80$; allowing an underestimate of the residual luminosity of $\sim 0.5 \mathrm{mag}$, as is common for faint sources, and with another $\sim 0.5 \mathrm{mag}$ of absorption, we obtain $\sim 40$. If indeed the residual feature marks the saddle point image, it is not possible to reproduce the positions of images and the required magnification ratio with a SIEMD (although this is not necessarily true for more complex models). Note that in this case absorption is probably less effective in boosting the magnification ratio, since the saddle point image is not so close to the galaxy core. 
We envisaged also the possibility that the object $\mathrm{C}$ is an additional image of the quasar. It seems that somehow this interpretation creates more problems than it solves. In fact the system size of $\sim 4.7^{\prime \prime}$ is excessive for a single galaxy lens. Furthermore, fitting object $\mathrm{C}$ in a configuration similar to that of Fig. 4, top panels, would require a reversed magnification ratio (A would be the most luminous image), very difficult to produce unless the mass is orthogonal to light. Finally, it is not clear if object $\mathrm{C}$ is truly point-like, since it seems to have a slightly smeared light profile, and the uncertainty on the PSF prevents us from a definite conclusion in this sense.

In the three-image configuration, the elongation of the quasar has to be of instrumental origin, as is explained in the following subsection.

\subsection{Quasar ellipticity}

The first, most obvious, explanation for the ellipticity of Q00453337 in the NACO image is instrumental, i.e. due to adaptive optics PSF deformation (see Sect. 3). As noted by Falomo et al. (2005), Q0045-3337 is elongated in a direction almost aligned to the vector radius of the guide star, but is not equal (with a difference of 10-15 degrees). The galaxy itself, and its brightest core in particular, do not appear to suffer similar deformation, but since their intrinsic brightness distribution is not radially symmetric, no definite conclusion can be drawn. If the ellipticity is real and not an instrumental artifact, a gravitational lensing effect can be invoked. In principle, image deformation can be produced by lensing of resolved sources, since in that case lensing conserves surface brightness, stretching the sources in the tangential directions and creating the well known arclets. However, it seems unlikely that this can be the case for Q0045-3337. In fact, while we stress again that the PSF in an adaptive optical image can be intrinsically complex, a magnified point source superimposed to a tangentially stretched extended source (like a QSO with its host galaxy) should result in a sharp bright core with a faint extended wing, i.e. the image should appear more elliptical at low brightness, and less at the brightness peak, where it should be dominated by the point source. This is exactly the opposite of what we see for Q0045-3337 (see the contour map of Fig. 2). The only way in which gravitational lensing can produce such behavior is by means of merging two or more images, unresolved by the NACO observation (note that the merging of two equal circular PSFs can produce an elliptical PSF with stronger ellipticity at the peak and weaker at the periphery). In the following section we explore this possibility in more detail.

\subsection{Five images}

If we require a lensing explanation to the quasar ellipticity, we need to produce five images. This can be obtained in any model with two at least partially nested caustic curves. For our purpose, we want the merging of two (or even three) images near to the tangential critical curve, in such a way that the NACO observation is not sufficient to resolve the contribution of the different images (as in Fig. 4, bottom panels). Images so close to a critical curve are strongly magnified. This can provide an explanation for the absence of the other images in the NACO observation based solely on strong lensing.

In particular, it is crucial to explain the non-detection of a minimum image, that is foreseen quite far away from the galaxy and therefore cannot be obscured. A conservative view assumes that the missing minimum image is fully undisturbed and therefore the full limit of the plate can be reached. We estimated a plate limit of 22.8 using the barely visible 22.6 residual object. In that case the obtained magnification ratio between the merged images and the missing minimum image is $\sim 100$. A less dramatic magnification ratio is allowed taking into account absorption and the effect of residual galaxy luminosity, and again an underestimate of object D magnitude. We made an educated guess of a $\sim 40$ magnification ratio. Note that the centroids of the merged images have to be very close each other; to give an order of magnitude example, the plate scale of the NACO image is only $0.054^{\prime \prime}$, and the distance between the two components used to fit the QSO with GALFIT in Sect. 3 is $0.052^{\prime \prime}$.

We verified the viability of the five-image scenario numerically with Gravlens simulations.

It is possible to obtain such a configuration with a SIEMD under two conditions: first, the tangential critical line must cross the position of the merged images (thus setting the galaxy mass scale), second, the source needs to be very close to the astroid caustic fold (Fig. 5). Assuming the first condition is fulfilled, like in Model I, the source plane area allowed by the second condition is very small. This result can be improved using different models of mass distribution. In particular, we employed an highly flattened distribution (so as to obtain a naked cusp geometry), as for the exponential disk mass distribution of Model IV.

Although such a strong flattening can hardly represent the observational parameters that we have, the simulation proves that in principle this model can more easily produce five images with the required magnification ratio.

\subsection{Five images versus three images}

We evaluated the relative probability of the five-image scenario with respect to the three-image scenario statistically. We chose a galaxy mass model able to reproduce either the three or the five images changing the source position. Therefore, the relative probability of the two scenarios can be evaluated as a ratio of weighted cross-sections, where the weight is needed to take into account properly the magnification bias. We simulated one million source positions for model I with the mock1 GravLens command (Fig. 5). We then picked up only the realizations able to exceed a fixed magnification ratio threshold, and we integrated the area spanned, weighting each source realization with its own magnification bias (e.g. in the integrand). For the three-image scenario we used a 20 threshold of magnification ratio, defined as the ratio between the fluxes of the two brightest images. For the five-image scenario, we defined the magnification ratio as the sum of the fluxes of the two brightest images over the flux of the third brightest image, and we chose the (optimistic) value of 40 for the threshold. As quasar number counts function we used a power law with two different values of the index, 1.85 and 2.23 (taken from the literature, e.g. Richards et al. 2005). The relative probabilities obtained are respectively $2.44 \%$ and $13.2 \%$. As expected (it appears as an exponent) the impact of the index of the quasar number counts function is very strong. Such probability values are not sufficiently low to firmly exclude the five-image scenario on the basis of this calculation alone. In fact while sources producing the five-image configuration are rare (Fig. 5), their overall magnification is large, so the inclusion of the magnification bias strongly enhances their weight.

The relative probability of the three- and five-image cases does not take into account the distinction between drops and mouth configurations; in fact to evaluate such a probability as a ratio between weighted cross-sections, we must make the hypothesis that the galaxy models are fixed; otherwise, we should 
evaluate the relative probability of the different galaxy models, something that goes beyond the scope of this paper. However, it is important to make clear that while the five images case requires strictly such a (rather large) value of the Einstein radius parameter, since the merged images are on the two sides of the tangential critical curve, with the three images a wider range of values is allowed. In particular, in the mouth configuration, it is possible to reproduce the observational constraints with a much lower value of the Einstein radius parameter. This smaller Einstein radius would be favored both by the SIS/Tully-Fisher evaluation of Sect. 4, and by the different normalizations proposed for application of the SIEMD to disk galaxies (e.g. Keeton \& Kochanek 1998). Furthermore, the mouth configuration leaves much more room for explaining the absence of the saddle point image, since it puts it much closer to the galaxy core.

\section{Discussion}

We performed two-dimensional photometry on the galaxy near to Q0045-3337. We confirmed its spiral nature and found evidence for two components with different orientations. We found a residual image after model subtraction, which is most likely due to a spiral arm, even if more interesting possibilities cannot be excluded. We then verified that no unusual parameters for the galaxy are required to produce, or not produce, image splitting. We also found some very simple strong lensing configuration capable of not contradicting existing data, either assuming a) that no other image is seen and Q0045-3337 observed elongation is instrumental, b) that the residual image is truly another lensed image of Q0045-3337, and the elongation is still instrumental, c) that Q0045-3337 elongation is due to the merging of two or more images, and no other image is seen. We evaluated the likelihood of the different cases, finding that case a) is the most probable, even if cases b) and c) cannot be rejected.

All these speculations can be easily verified observationally. In particular, another optically adaptive image with more information on the PSF behavior would confirm or rule out the ellipticity of Q0045-3337 and would also verify if object C is point-like. A measurement of the galaxy redshift, while probably not conclusive, would help to constrain how likely the production of multiple images is. Deeper pointings could reveal additional images or put tighter constraints on required magnification ratios. If case c) turns out to be the most probable, an HST image could resolve the merged images. At present, strong lensing effects on Q0045-3337 can only be labeled as possible. Spiral strong lenses are rare objects. The current view assumes that the cross-section for image splitting is not generally increased by the presence of a thin massive disk, at least once corrected for the inclination and once properly taken in account the current observational capabilities; furthermore, absorption biases strongly against the detection (Bartelmann \& Loeb 1998; Keeton \& Kochanek 1998; Perna et al. 1997). In such a context, the galaxy close to Q0045-3337 can offer a valuable contribution to our knowledge of spiral galaxy mass distributions and of lensing statistics. Even in the case without image splitting, as it is likely both for the sample of Narayan \& Schneider (1990) and for the Lyman absorbers of Le Brun et al. (2000), interesting upper limits can be placed on the galaxy mass inside the Einstein radius (once the redshift is known). Furthermore, the lensing effect of stretching of resolved sources could make Q0045-337 a valuable target again for deeper host galaxy studies (as was originally selected by Falomo et al. 2005). As a final comment, it is interesting to note that this case can be a perfect example of advantages and drawbacks of adaptive optics applied to strong lensing.

Over the last few years, HST has been the principal instrument in strong lensing studies, and it is interesting to evaluate if it can be - at least partially - replaced by adaptive optics earth telescopes, especially in the case of a gap between the end of HST operations and the start of the James Webb Space Telescope operations. As Q0045-3337 and its neighbor galaxy tell us, a necessary condition to be fulfilled in this case is a correct PSF evaluation observational procedure (as was initially proposed for Q0045-3337); otherwise, the unresolved uncertainty between real and instrumental deformations hampers the exploitation of the optimal resolution for the purpose of lens studies.

Acknowledgements. We thank Aldo Treves and Renato Falomo for pointing out to us the existence of Q0045-3337, for useful discussions on lensing or instrumental origin of the observed deformation, and for kindly sharing with us the NAOS-CONICA ESO VLT observational data. We thank the referee, Neal Jackson, for his substantial contribution to the improvement of the paper. Marco Miranda is supported by the Swiss National Science Foundation.

\section{References}

Adelman-McCarthy, J., Agueros, M. A., Allam, S. S., et al. 2006, ApJS, 162, 38 Bartelmann, M., \& Loeb, A. 1998, ApJ, 50348

Browne, I. W. A., Wilkinson, P. N., Jackson, N. J. F., et al. 2003, MNRAS, 341, 13

Clénet, Y., et al. 2006, in Advances in Adaptive Optics II, ed. B. L. Ellerbroek, \& D. Bonaccini Calia, Proc. SPIE, 6272, 62723T

Falomo, R., Kotilainen, J. K., Scarpa, R., \& Treves, A. 2005, A\&A, 434, 469

Huchra, J., Gorenstein, M., Kent, S., et al. 1985, AJ, 90, 691

Iovino, A., Clowes, R., \& Shaver, P. 1996, A\&AS, 119, 265

Jackson, N., de Bruyn, A. G., Myers, S., et al. 1995, MNRAS, 274, L25

Kassiola, A., \& Kovner, I. 1993, ApJ, 417, 450

Keeton, C. R. 2001, ApJ, submitted [arXiv: astro-ph/0102340]

Keeton, C. R., \& Kochanek, C. S. 1998, ApJ, 495, 157

Keeton, C. R., Kuhlen, M., \& Haiman, Z. 2005, ApJ, 621, 559

Kochanek, C. S., Schneider, P., \& Wambsganss, J. 2004, Part 2 of Gravitational Lensing: Strong, Weak \& Micro, Proceedings of the 33rd Saas-Fee Advanced Course, ed. G. Meylan, P. Jetzer, \& P. North (Berlin: SpringerVerlag)

Kormann, R., Schneider, P., \& Bartelmann, M. 1994, A\&A, 284, 285

Le Brun, V., Smette, A., Surdej, J., \& Claeskens, J.-F. 2000, A\&A, 363, L837

Myers, S. T., Jackson, N. J., Browne, I. W. A., et al. 2003, MNRAS, 341, 1

Narayan, R., \& Schneider, P. 1990, MNRAS, 243, 192

Patnaik, A. R., Browne, I. W. A., King, L. J., et al. 1993, MNRAS, 261, 435

Peng, C. Y., Ho, L. C., Impey, C. D., \& Rix, H.-W. 2002, AJ, 124, 266

Perna, R., Loeb, A., \& Bartelmann, M. 1997, ApJ, 488, 550

Pierini, D., \& Tuffs, R. J. 1999, A\&A, 343, 751

Pramesh Rao, A., \& Subrahmanyan, R. 1988, MNRAS, 231, 229

Richards, G. T., Croom, S. M., Anderson, S. F., et al. 2005, MNRAS, 360, 839

Saha, P., \& Williams, L. L. R. 2003, AJ, 125, 2769

Schramm, T. 1990, A\&A, 231, 19

Véron-Cetty, M. P., \& Véron, P. 2001, A\&A, 374, 92

Winn, J. N., Hall, P. B., \& Schechter, P. L. 2003, ApJ, 597, 672

White, R. L., Becker, R. H., Helfand, D. J., et al. 1997, ApJ, 475, 479

York, D. G., Adelman, J., Anderson, J. E., et al. 2000, AJ, 120, 1579 